BONPLANDIA 19(1): 79-89. 2010

\title{
MALVACEAE VARIA
}

\author{
ANTONIO KRAPOVICKAS
}

Summary. Krapovickas, A. 2010. Malvaceae varia. Bonplandia 19(1): 79-89. ISSN: 05240476.

The new species Acaulimalva pazensis, Pavonia pilifera, Sida Beckii and S. yungasensis are described. The new name Pavonia Arechavaletana is proposed for P.urticifolia Arechav. and Sida planicaulis is mentioned for the first time for Argentina.

Key words: Taxonomy, Acaulimalva, Pavonia, Sida.

Resumen. Krapovickas, A. 2010. Malvaceae varia. Bonplandia 19(1): 79-89. ISSN: 05240476.

Se describen las especies nuevas Acaulimalva pazensis, Pavonia pilifera, Sida Beckii y S. yungasensis, se propone el nuevo nombre Pavonia Arechavaletana para P.urticifolia Arechav. y se cita por primera vez a Sida planicaulis para Argentina.

Palabras clave: Taxonomía, Acaulinalva, Pavonia, Sida.

\section{Acaulimalva pazensis Krapov., sp. nov.}

Fig. 1

Fruticulus depressus, folia rosulata, petiolus marginibus ciliato, lamina ovata, glabra, marginibus crenatis-serratis. Flores singuli, pedunculo 5-10 $\mathrm{mm}$ longo. Bracteolae lanceolatae, glabris. Calyx $9 \mathrm{~mm}$ longis, glaber, segmentis extra setosus. Corolla albo lilacea, petala 6-8 $\mathrm{mm}$ longa, $2 \mathrm{~mm}$ lata. Stamina numeerosa, in caput globosum instructa. Styli ca. 8, stigmata capitata. Carpella dorso paullo verrucosa, apice laeve, tomentosa.

Typus: Bolivia. La Paz, Prov. Murillo, La Paz Calacoto, 18 km hacia Collana, 3800 m. Laderas altas sobre el valle del río, con chacras, pastizales y arbustos al borde. Perenne, corola blanca-rosada. 3-IV-1988. S.G. Beck 13787 (holotypus LPB, isotypus: CTES).
Hierba perenne, acaule, arrosetada. Raiz 10 $\mathrm{mm}$ de diámetro. Estípulas $6 \mathrm{~mm}$ long. x 1-2 mm lat, agudas, castañas, glabras, con el margen ciliado. Pecíolo 10-25 mm long., ambas caras glabras, margen ciliado. Lámina ovada, 20-25 mm long. x 15-20 mm lat., margen crenado-aserrado, ambas caras glabras. Flores solitarias, agrupadas en el centro de la planta. Pedúnculo 5-10 mm long., glabro. Bractéolas $2 \mathrm{~mm}$ long. lanceoladas, glabras. Cáliz $9 \mathrm{~mm}$ long., glabro, lóbulos triangulares $5 \mathrm{~mm}$ long. x $3 \mathrm{~mm}$ lat. Corola blanca-rosada. Pétalos 20 $\mathrm{mm}$ long. Tubo estaminal mas breve que la corola, con pelos simples hialinos esparcidos. Carpelos 15-20, uniovulados. Mericarpos 2,5 $\mathrm{mm}$ long. x $2 \mathrm{~mm}$ lat. lisos, dehiscentes, con una pequeña arista apical, cara superior con pelos simples ca. $1 \mathrm{~mm}$ long.

Distribución geográfica: Vive en los cerros al sur de la ciudad de La Paz, en la cuenca del río

${ }^{1}$ Instituto de Botánica del Nordeste, Casilla de Correo 209, 3400 Corrientes, Argentina. 


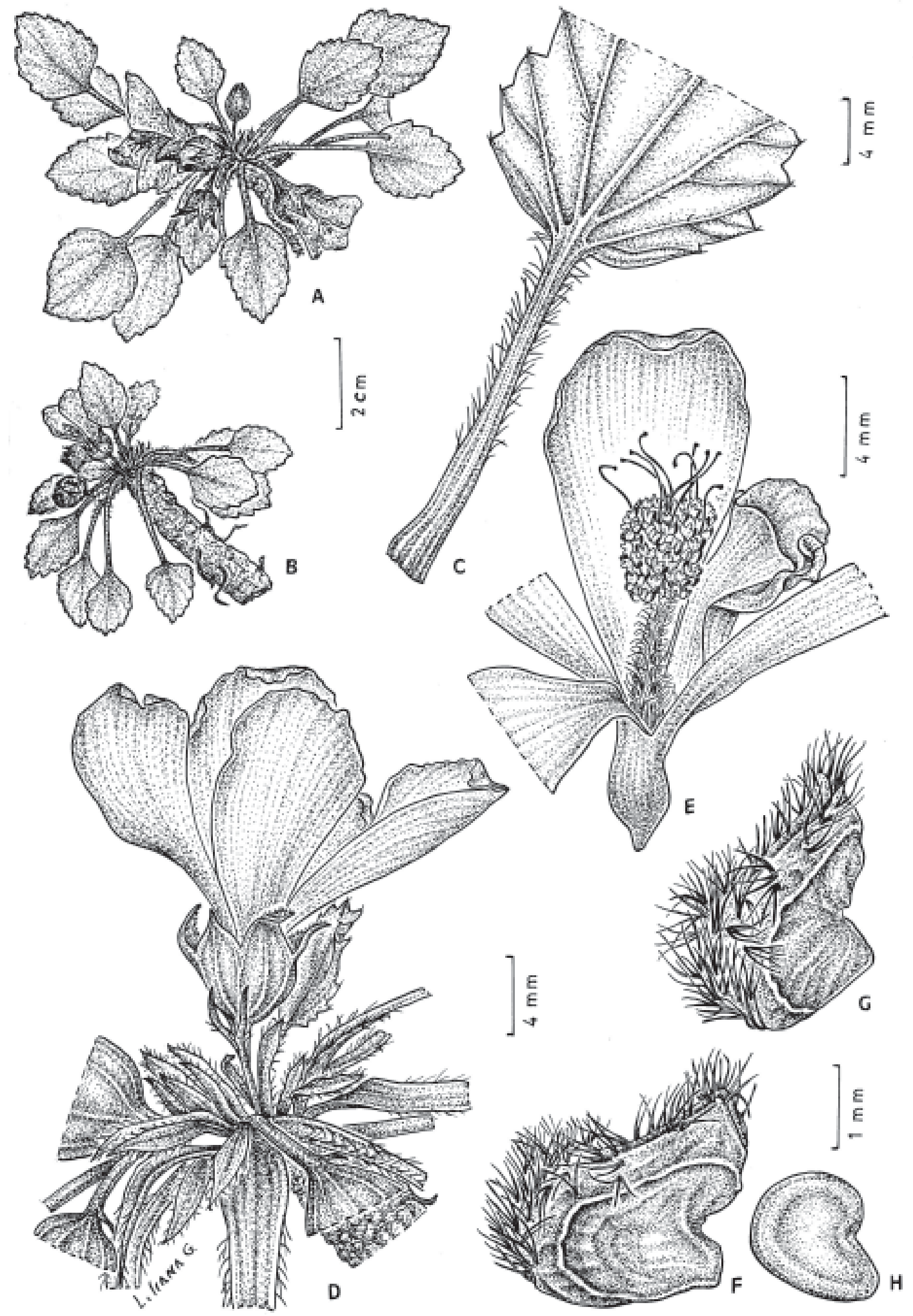

Fig. 1. Acaulimalva pazensis. A: planta. B: planta vista lateral. C: pecíolo y base de la hoja. D: flor. E: flor y tubo estaminal. F: mericarpo, cara lateral. G: mericarpo, cara dorsal. H: semilla (A-H, Beck 13787). 
de La Paz, entre 3800 y 4250 m de altutura.

Etimología: El nombre específico se debe a que vive en la cuenca del río de $\mathrm{La} \mathrm{Paz}$, no lejos al sur de la ciudad de La Paz, capital oficial de la República de Bolivia.
Paratypi: BOLIVIA. La Paz: Prov. Murillo, an der Strasse nach Nunumayumi, linke Talseite des Rio de La Paz, Mecapaca, 3950 m, 29-III-1981, Feuerer 5725 b (LPB, CTES); Cerro Cuñamani, 4250 m, 3-IV-1982, Ruthsatz 1716 (CTES).

\section{Las especies de Acaulimalva de Bolivia (Krapovickas, 1974) se pueden distinguir por medio de la siguiente clave}

A. Lámina de las hojas glabra Cáliz glabro.

B. Pedúnculo de la flor 10-40 mm long. Mericarpos dehiscentes en el ápice. Hojas pinatilobadas.

C. Mericarpos rugosos en el dorso. Carpelos ca. 20. Pedúnculo 40 mm long. Cochabamba.

A. Steinbachii Krapov.

C’ Mericarpos lisos. Pedúnculo 10-20 mm long. Carpelos 15. Chuquisaca, Potosí.

A. oriastrum (Wedd.) Krapov.

B’ Pedúnculo 5-10 mm long. Mericarpos totalmente dehiscentes, lisos. Carpelos 15-20. Hojas enteras, margen crenado. La Paz.

A. pazensis Krapov.

A' Hojas pilosas. Mericarpos indehiscentes, con dos costillas dorsales dentadas

D. Pedúnculo 20 mm long. Cáliz estrigoso. Carpelos 8-9. Sucre, La Paz.

A. dryadifolia (Solms) Krapov.

D’ Pedúnculo 10-90 mm, Cáliz glabro. Carpelos 7-13. La Paz, Potosí, Cochabamba.

A. nubigena (Walp.) Krapov.

\section{Pavonia Arechavaletana Krapov. \& Fryxell, nom. nov.}

Iconografía: Fryxell, 1999: 219, fig. 82.

Pavonia urticifolia Arechav., Anal. Mus. Nac. Montevideo 3: 144. 1898. Typus: Uruguay. «Orillas de montes o selvas, en Tacuarembó». Arechavaleta s.n. (holotypus MVM!). (non Pavonia urticifolia C.Presl, 1835).

Pavonia ramosissima (Arechav.) Fryxell \& Krapov., Fl. Neotrop. 76: 218, figs. 48 \& 82. 1999 (excluído el basónimo Pavonia sepium var. ramosissima Arechav. 1898).

Arbusto 0,5-3 m alt, Tallos densamente estrellado pubescente. Estípulas 5-8 mm long., filiformes. Pecíolo 3-12 mm long. estrellado pubescente. Lámina oval-elíptica, hasta 12 x $5 \mathrm{~mm}$, base truncada, ápice agudo, margen aserrado, ambas caras estrellado pubescentes.
Flores axilares pero con frecuencia agrupadas formando una inflorescencia apical congesta. Pedicelos 0,5-3 cm long. Bractéolas 5-6, lanceoladas, $7 \mathrm{~mm}$ long. $\mathrm{x}<1 \mathrm{~mm}$ lat., lanceoladas. Cáliz $5 \mathrm{~mm}$ long., ciliado. Pétalos amarillos, 11-20 mm long., cara externa estrellado pubescente. Tubo estaminal 10-11 mm long., glabro. Estigmas 4-5 mm más largos que el tubo estaminal. Mericarpos con 3 esoinas, la central erecta y las laterales divergentes . espinas con pelos retrorsos. Cromosomas 2n=56 (Krapovickas 41933).

Distribución geográfica: Sur de Brasil. NE de Argentina y $\mathrm{N}$ de Uruguay. Vive en el borde de bosques y selvas, al contrario de $P$. sepium que crece exclusivamente en el interior del bosque.

Obs.: Posteriormente a la publicación de Pavonia ramosissima (Fryxell, 1999) tuve 
oportunidad de estudiar en el Museo Nacional de Montevideo los holotipos de Pavonia urticifolia Arechav. y de P. sepium var. ramosissima Arechav., comprobando que nuestra interpretación de las descripciones originales estaba errada. El tipo de Pavonia sepium var. ramosissima tiene hojas con domacios en el hipofilo, es subglabro y tiene flores solitarias, por lo cual es un sinónimo de Pavonia sepium A.St.-Hil. En cambio P. urticifolia carece de dichos domacios, es estrellado-tomentosa y tiene flores solitarias, con frecuencia agrupadas en el ápice de las ramas formando una inflorescencia aglomerada, lo cual la diferencia facilmente de $P$. sepium.

Material estudiado. ARGENTINA. Misiones: Dep. Gral. Manuel Belgrano, Bernardo de Irigoyen, 791 m, 20-II-2008, Keller \& Prance 5082 (CTES). BRASIL. Paraná: Quatro Barras, 6-IV-1971, Dombrowski 3384 (CTES,MBM); Santa Maria do Oeste, 19-II-2004, Hatgschbach \& al. 76843 (CTES,MBM); Mun. Colombo, Bacaitava, 10-II-1961, Hatschbach 7781 (CTES,MBM); Ressaca, 14-II-1975, Kummrow 886 (CTES, MBM); Mun. Paulo Fronin, Vicinal Nove, 28-I-2005, Ribas \& al. 6612 (CTES,
MBM); Mun. Rio Branco do Sul, Madre, 26-I1961, Hatschbach 7666 (CTES, MBM); Itaperuçú, 14-II-19676, Dombrowski 2447 (CTES, MBM). Rio Grande do Sul: Cruz Altinha, BR 285, Km 130, 29-XI-1980, Krapovickas \& Vanni 37049 (CTES, HAS); S. Fco. de Paula, Vila Oliva, 6-I-1946, Rambo 31098 (CTES, LIL); Planalto, 26-III-1987, Bueno 4993 (CTES, HAS); Mun. Caxias do Sul, 30-I-1994, Pedersen 15929 (C, CTES, TEX); Mun. Farroupilha, 28-XII-1977, Aguiar \& Martau 5523 (CTES, HAS); Parque dos Pinheiros, 23-V-1978, Bueno 684 (CTES, HAS); Mun. Lagoa Vermelha, BR 285, Km 61, Krapovickas \& Cristóbal 41933 (C, CTES, F, G, GH, HAS, MBM, MO, NY). Santa Catarina: Palmares, Campos Novos, 800 m. 31-I-1963, Reitz 6439 (CTES); Taquara Verde, 800 m, Reitz \& Klein 11769 (CTES); 16 km de BR-116, camino a Campo Belo do Sul, 14-I-1988, Krapovickas \& Cristóbal 41973 (CTES); Fraiburgo, 22-I-1992, Krapovickas \& Cristóbal 43972 (CTES); Parque das Pedras Brancas, 10 km SE de Lages, 17-I-1988, Krapovickas \& Cristóbal 42079 (CTES); $13 \mathrm{~km}$ de Painel, camino a Urupema, 11-I-1988, Krapovickas \& Cristóbal 41964 (CTES); Mun. Urubici, 6 km N de Pericó, 25XII-1982, Krapovickas \& Schinini 38303 (CTES). URUGUAY. Tacuarembó: Valle Edén, 20-II-1987, Izaguirre \& al. 18697 (CTES,MVFA).

\section{Las especies afines con flores amarillas, pertenecientes al subgénero Typhalaea sect. Urenoideae (Fryxell, 1999: 205) se pueden distinguir por medio de la siguiente clave}

A. Hojas con domacios (un manojo denso de pelos en las axilas del nervio principal y de los nervios secundarios en el hipofilo).

B. Pétalos 8-15 mm long. Espinas laterales de los mericarpos radiadas. 2n=56. S de $20^{\circ} \mathrm{S}$ en América del Sur.

P. sepium A.St.-Hil.

B'. Pétalos 20-28 mm long. Espinas laterales de los mericarpos erectas. Sierras subandinas de Bolivia y NW de Argentina.

P. formosa Fryxell

A’. Hojas sin domacios.

C. Hierba rastreras, radicantes en los nudos,

D. Hoja ovada, más larga que ancha. Brazil (MG, SC, RS) y Argentina (Misiones).

P. flavispina Miquel

D’. Hoja reniforme o suborbicular. Brasil (S.Catarina).

P. renifolia Krapov. 
C'. Arbustos o subarbustos erectos, no radicantes.

E. Pedicelos de la flor 1-2 cm long. Cáliz 8-11 mm long. Tubo estaminal $20 \mathrm{~mm}$. Estigmas igual a la corola o exsertos. Antillas, Florida.

P. spinifex (L.) Cav.

E’. Pedicelos hasta $6010 \mathrm{~cm}$. Estigmas más cortos que la corola. Tubo estaminal $<11 \mathrm{~mm}$.

F. Pedicelos 10 cm. Pétalos 10 x3 mm. Brasil (Paraná, S. Catarina).

P. stenopetala Krapov.

F’. Pedicelos $<6 \mathrm{~cm}$. Pétalos $>10 \mathrm{~mm}$ y más anchos

G. Espinas laterales de los mericarpos erectas. Cuencas de los ríos Paraguay y Paraná en Paraguay y Argentina.

G’. Espinas laterales radiadas.

P. Narcisii Krapov.

H. Espinas de los mericarpos subglabras, hacia el ápice presentan pelos retrorsos diminutos.

I. Cáliz pentacarinado, más corto que el calículo, con lóbulos angostos, 2 mm lat. y rígidos. Bolivia, W de Santa Cruz.

P. ionnthacarpa Krapov.

I’. Cáliz liso, más largo que el calículo, lóbulos > 2 mm lat. Brasil, E de S.Catarina.

P. horrida Krapov.

H'. Espinas de los mericarpos con pelos retrorsos manifiestos.

J. Tubo estaminal 10-11 mm long.

K. Flores solitarias. 2n=112. Perú y Brasil hasta NE de Argentina y N de Uruguay.

P. communis A.St.-Hil.

$K$ '. Flores con frecuencia agrupadas en una inflorescencia apical congesta. $2 n=56$. NE de Argentina, S de Brasil y $\mathrm{N}$ de Uruguay.

P. Arrechavaletana Krapov. \& Fryxell

J'. Tubo estaminal $<7 \mathrm{~mm}$

L. Hoja angosto-elíptica u oblonga, mas ancha en el medio, relación largo/ancho 2,54. E de México.

P. uniflora (Sessè \& Mociño) Fryxell

L'. Hoja ovado-lanceolada u ovado-elíptica, más ancha por debajo del medio, relación largo/ancho de la hoja ca.2. NW de América del Sur.

P. sepioides Fryxell

\section{Pavonia pilifera Krapov., sp. nov.}

Fig. 2

Sufrutex ca. 2 m altus. Rami hirsuti, trichomatibus longis, simplicibus vel stellatis 2-3 mm longis. Stipulis linearibus 4-5 cm longis. Laminis foliorum ellipticis, penninervibus, margine crenatus, supra et infra pilis simplicibus $2 \mathrm{~mm}$ longis. Pedicellis axilaribus solitariis, $20-25 \mathrm{~cm}$ longis. Bracteis involucellorum 7 ovalis, $25 \mathrm{~mm} \times 5-6 \mathrm{~mm}$. Petalis albis, quam calycis subaequantes.

Typus: Brasil. Bahia, Mun. Ibirapitanga, Faz. Palmeiras. Subarbusto de ca. $2 \mathrm{~m}$ de altura; caule piloso; estípulas filiformes, pelosas; folhas pilosas, discolores, cartáceas, alternas; flores solitarias, corola blanca, estambres amarelos, látex transparente. Ocasional. Floresta Ombrófila Densa. 17-II-1998, A. S. Conceição 216 (holotypus HRB, isotypus CTES).

Subarbusto $2 \mathrm{~m}$ alt. Tallo hirsuto, con pe- 


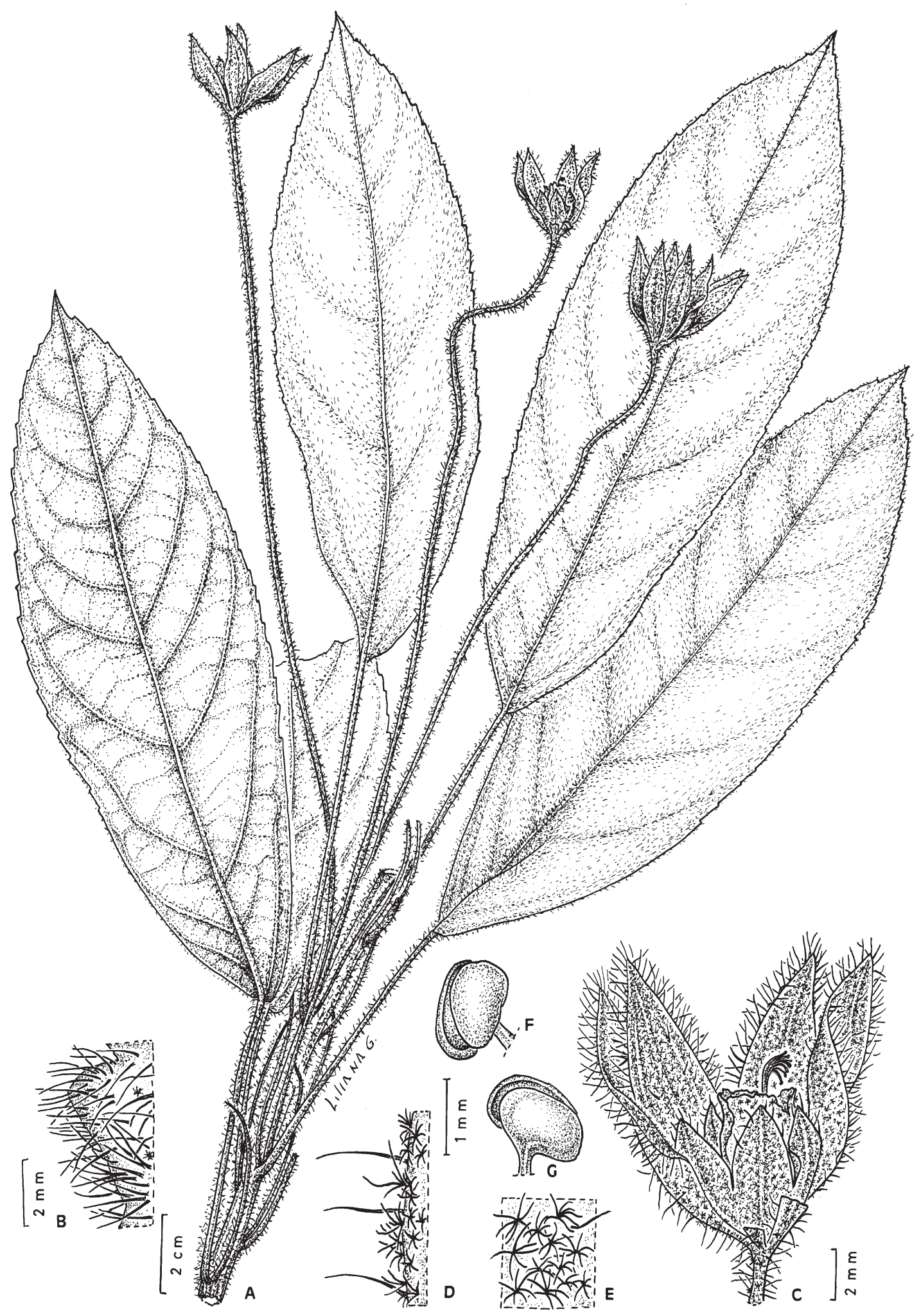

Fig. 2. Pavonia pilifera. A: rama. B: indumento de la hoja. C: flor. D: indumento de las bractéolas. E: indumento del cáliz. F-G: anteras (A-G, Conceiçao 216). 
los simples o con as ramas,2-3 mm long. Estípulas 3-5 cm long., lineales, pilosas. Pecíolo 6-7 cm long. piloso, con pelos similares a los del tallo. Lámina elíptica, $16-20 \mathrm{~cm}$ long. x 5-6 cm lat. ápice agudo, margen levemente crenado; ambas caras con pelos con pelos simples, rígidos, $2 \mathrm{~mm}$ long., antrorsos, hipofilo con nervios sobresalientes, pero el central más destacado. Flor solitaria. Pedicelo 20-25 cm long. con pelos simples como los de la lámina foliar. Calículo de 7 bractéolas 25 mm long x 5-6 mm lat. con pelos estrellados diminutos, esparcidos, margen ciliado con pelos simples $1 \mathrm{~mm}$ long., cara interna con pelos estrellados diminutos, esparcidos. Cáliz 10$12 \mathrm{~mm}$ long., cubierto completamente de pelos estrellados amarillentos, lóbulos $9 \mathrm{~mm}$ long. x $4 \mathrm{~mm}$ lat. Corola blanca, apenas más larga que el cáliz. Estilo sobrepasa $5 \mathrm{~mm}$ a la corola, estigmas 9.

Distribución geográfica: Conocida hasta ahora sólo de la localidad tipo.

Etimología: El nombre específico deriva del latín: pilis $=$ pelo y fere $=$ llevar.

Obs. 1: Pavonia pilifera pertenece al subgénero Goetheoides (Gürke) Ulbr. por sus hojas elípticas, grandes, no lobadas y con el nervio medio sobresaliente en el hipofilo (Fryxell, 1999, Krapovickas 1982, 1990). Con esta nueva especie se eleva a 28 los integrantes del subgénero Goetheoides. Número bastante elevado por ser este grupo endémico de la mata atlántica en los estados de Pernambuco, Bahia. Espirito Santo y Rio de Janeiro (Fryxell, 1999, 2009).

Obs. 2: Son tres las especies de este subgénero con tallo hirsuto, con pelos en su mayoría simples, $2 \mathrm{~mm}$ long. De éstas, Pavonia ovaliphylla G.E.Esteves \& Krapov. y P. atlantica Fryxell tienen las hojas glabras. En cambio P. ciliata G.L.Esteves \& Krapov. tiene el tallo hirsuto y las hojas con pelos simples, antrorsos como en $P$. pilifera (Esteves \& Krapovickas, 1995). Ambas especies se diferencian porque $P$. ciliata tiene el calículo con 14-16 bractéolas lineares dispuestas en espiral (secc. Tricalycares Gürke), en cambio $P$. pilifera tiene 7 bractéolas 5-6 $\mathrm{mm}$ lat. dispuestas en un solo verticilo (secc. Goetheoides).

\section{Sida Beckii Krapov., sp. nov.}

Fig. 3

Sufrutex $1 \mathrm{~m}$ altus. Foliis breviter petiolatis. Lamina rhombeo-lanceolata, serrata, basim versus integerrima,usque ad $9 \mathrm{~cm}$ longa, $3 \mathrm{~cm}$ lata, pauci pilis stellatis punctiformis. Floribus solitariis, longe pedunculatis. Calyce nervis 10 basi noduloso-incrassatis, pilis stellatis brevis. Tubus stamineus $2 \mathrm{~mm}$ long. pilosus. Carpidiis ca. 10, biaristatis.

Typus. Bolivia. Cochabamba, Prov. José Carrasco Torrico. Camino a Villa Tunari. El Sillar, derrumbe grande con pérdida de la plataforma ( $\mathrm{km} 389)$. $17^{\circ} 04^{\prime} 32$ 'S, 654' W. Alt. $800 \mathrm{~m}$. Restos de bosque submontano. Arbusto $1 \mathrm{~m}$, hojas lanceoladas, flores amarillas-anaranjadas. 8-I-2009. S. G. Beck 33051 (holotypus LPB, isotypus CTES).

Subarbusto $1 \mathrm{~m}$ alt. Tallo con pelos estrellados diminutos, ralos. Estípulas filiformes 5-6 mm long., con pocos pelos estrellados punctiformes. Pecíolo 4-5 mm long., con una pequeña protuberancia apenas dentada en la base, con pocos pelos estrellados punctiformes y una línea densa de pelos estrellados blancos, mayores, en el dorso. Lámina rómbico-lanceolada, hasta $9 \mathrm{~cm}$ long. x $2 \mathrm{~cm}$ lat., margen liso en los $2 \mathrm{cms}$ basales y el resto aserrado; haz con nervaduras rojizas, con muy pocos pelos estrellados punctiformes, algo más densos sobre las nervaduras; envés con nervaduras verde claro, subglabro. Flores solitarias. Pedúnculo 35-45 mm long., articulado $\pm 10 \mathrm{~mm}$ por debajo del cáliz. Cáliz $5 \mathrm{~mm}$ long., con 10 costillas sobresalientes en la base, con pelos estrellados muy pequeños, lóbulos triangulares $3 \mathrm{~mm}$ long. x $3 \mathrm{~mm}$ lat. Corola amarillo-anaranjada. Tubo estaminal 2 $\mathrm{mm}$ long. con pelos estrellados. Mericapos ca. 10, $3 \mathrm{~mm}$ long. $\mathrm{x} 2 \mathrm{~mm}$ lat. y 2 aristas $1 \mathrm{~mm}$ long., glabros. Semilla glabra.

Distribución geográfica: Vive en la selva 


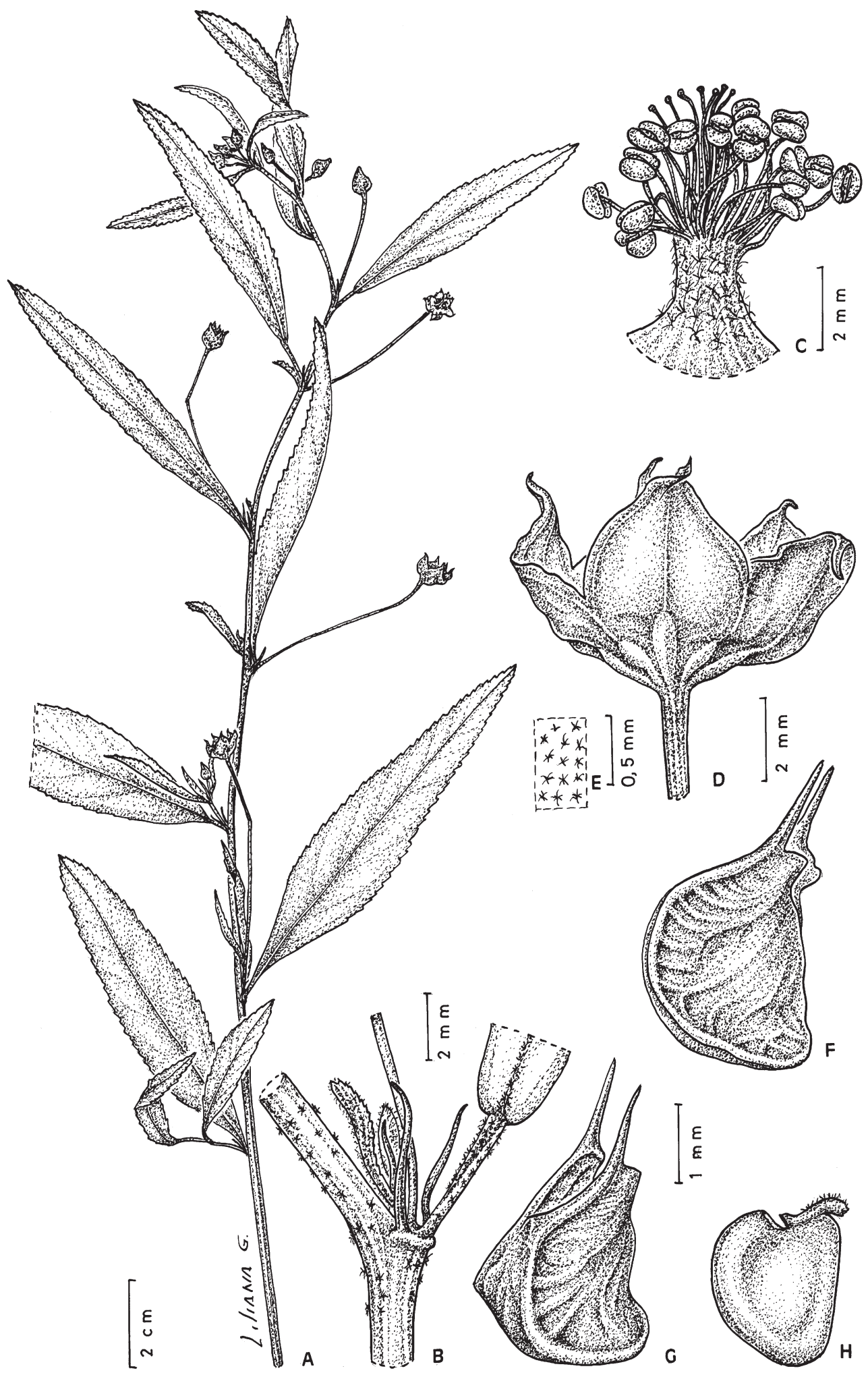

Fig. 3. Sida Beckii. A: rama. B: estípulas y pecíolo. C: tubo estaminal. D: cáliz. E: indumento del cáliz. F-G: mericarpos. H: semilla (A-H, Beck 33051). 
húmeda de los Yungas del Chapare, a $800 \mathrm{~m}$ s.n.m., en la ladera norte de la cordillera de Cochabamba, en Bolivia.

Etimología: Nombre dedicado a Stephen G. Beck, quién coleccionó el ejemplar tipo.

Obs.: Esta especie pertenece a la sección Sida por su hoja con el margen liso en la parte basal y por las costillas sobresalientes en la base del cáliz. Se diferencia de las especies sudamericanas de esta sección por tener ambas caras de la hoja subglabras, con pocos pelos estrellados punctiformes y por los mericarpos completamente glabros (Krapovickas, 2007).

\section{Sida planicaulis Cav.}

Cavanilles, A. J., Diss. Bot. Sida: 24. 1785.

Sida planicaulis es una especie casi exclusiva de Brasil donde crece «desde Pernambuco hasta Porto Alegre en Rio Grande do Sul, especialmente en zonas bajas a lo largo de la costa atlántica. se ha expandido a los océanos Índico y Pacífico, donde se la ha mencionado repetidas veces».

Posteriormente a la publicación de una revisión de Sida sect. Distichifolia (Monteiro) Krapov.(Krapovickas, 2003), S. planicaulis fué coleccionada en el NE de la provincia de Misiones, en Argentina. Las localidades más cercanas en Brasil son Tenente Portela en el NW de Rio Grande do Sul y Ponta Porá en Mato Grosso do Sul.

Material estudiado. ARGENTINA. Misiones: Dep. Guaraní, Colonia Pepirí, 19-X-2006, Keller \& Prance 3693 (CTES); Ruta Costera $n^{\circ}$ 2, acceso a complejo turístico Aldea Yabotí, 12-X 2006, Keller 3684 (CTES).

\section{Sida sección Nelavaga Borss. Waalk.}

Sida yungasensis Krapov., sp. nov.

Fig. 4

Herba $70 \mathrm{~cm}$ alta. Caulibus velutinus et pilis 2 mm longis indutis. Lamina ovata, acuta, basi cordata, supra et infra stellato-pubescens. Floribus axillaribus solitarii. Pedicellus 5-10 mm longus. Calyx $7 \mathrm{~mm}$ longus. Carpidiis 5, rotundatis, glabriis.

Typus: Bolivia, La Paz, Nor Yungas, Coroico, Bajando hacia Vagante sobre el río Santa Bárbara, 16010’34" S, 6701'78" W. Alt. 1400 m, 9-VII-2005, Beck 30444 (holotypus LPB, isotypus CTES).

Hierba erecta $70 \mathrm{~cm}$ alt. Tallo con una capa no muy densa de pelos estrellados muy pequeños y pelos glandulares y también con abundantes pelos simples, rígidos, $2 \mathrm{~mm}$ long. Estípulas $1 \mathrm{~mm}$ long., caducas. Pecíolo 5-15 mm long., con indumento similar al del tallo. Lámina ovada, base cordada, ápice agudo, 25 mm long. x 15 mm lat., margen crenado; ambas caras cubiertas totalmente de pelos estrellados muy pequeños, blanquecinos en el envés. Flores solitarias axilares, o en ramas breves. Pedúnculo 5-10 mm long., articulado en el tercio superior, con indumento similar al del tallo. Cáliz 7 mm long., lóbulos triangulares $4 \mathrm{~mm}$ long. $\mathrm{x} 3 \mathrm{~mm}$ lat., con indumento de pelos estrellados pequeños, pelos glandulares y setas $2 \mathrm{~mm}$ long. sobre los nervios y márgenes de lo lóbulos; margen de los lóbulos algo más oscuro. Corola anaranjada. Carpelos 5. Mericarpos redondos, negros, glabros, $2 \mathrm{~mm}$ long. x 1,5 m lat., indehiscentes.

Distribución geográfica: Conocida sólo de la región selvática de los Yungas de La Paz en Bolivia.

Obs.: Por tener 5 carpelos, hojas cordadas, agudas y cáliz con el margen de los lóbulos algo mas oscuro, Sida yungasensis pertenece a la sección Nelavaga Borss.Walk. (Fryxell, 1985, Krapovickas, 2006). Por sus mericarpos múticos, redondos, $S$. yungasensis es afín $S$. Luschnatiana, S. Martiana y S. Schininii (Krapovickas 2006).

Paratypus: BOLIVIA. La Paz: Nor Yungas: Rd. up Río Huarinilla for ca. 3 km (this being 5,4 km SW of Yolosa along rd. towards Chuspipata); 1280 m; са. 16²1's, 67047'W, 6V-1990, Luteyn \& Dorr 13513 (CTES, NY). 


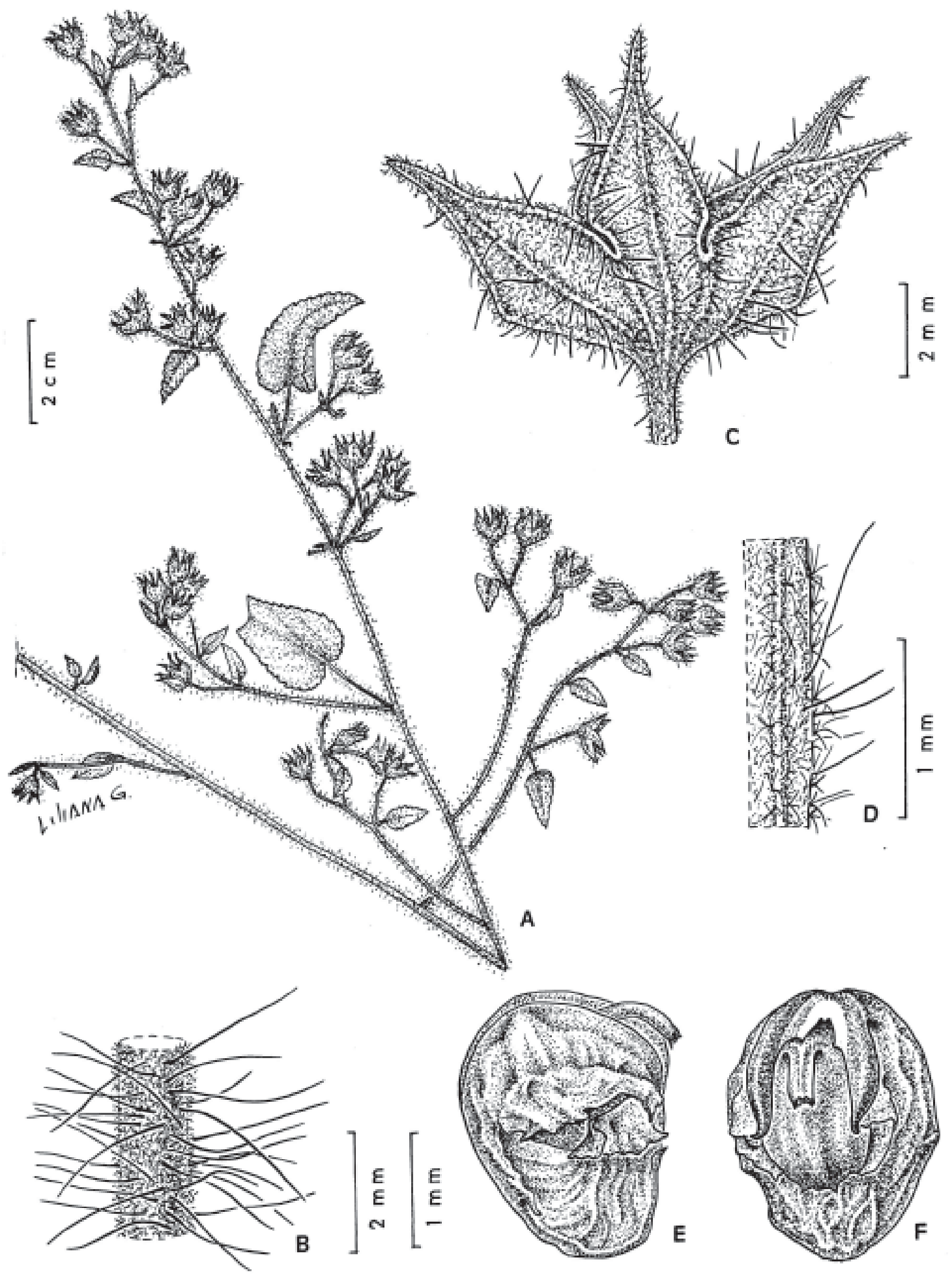

Fig. 4. Sida yungasensis. A: rama. B: indumento del tallo. C: cáliz. D: indumento del cáliz. E-F: mericarpos, vista dorsal y ventral (A-F, Beck 30444). 


\section{Estas especies con mercarpos redondos se pueden identificar por medio de la clave siguiente}

A. Tallo con pelos estrellados y pocos glandulares y pelos simples $2 \mathrm{~mm}$ long. Haz con pelos estrellados. Mericarpos múticos, glabros. Bolivia, Yungas de La Paz.

S. yungasensis Krapov.

A'. Tallo con pelos glandulares y pelos simples 1-3 mm long.

B. Haz con pelos glandulares pequeños. Mericarpos múticos, glabros. Centro de Brasil y Chiquitanía.

S. Luschnatiana Steud.

B'. Haz con pelos simples

C. Haz con pelos 0,5-1 mm long. Mericarpos múticos, con pelos muy pequeños en el ápice. Minas Gerais.

S. Martiana A.St.-Hil.

C’. Haz con pelos simples menores de 0,2 mm. Mericarpos con aristas 0,2 mm long., dorso y ápice pilosos. Chiquitanía y Mato Grosso do Sud.

S. Schininii Krapov.

\section{Agradecimientos}

Deseo expresar mi agradecimiento a Liliana Gómez por las ilustraciones que acompañan este texto.

\section{Bibliografía}

ESTEVES, G.L. \& A. KRAPOVICKAS. 1995. Duas novas espécies de Pavonia Cav. (Malvaceae) do sul da Bahia, Brasil. Hoehnea 22: 41-45.

FRYXELL, P.A. 1985. Sidus sidarum V. The North and Central American species of Sida. Sida 11(1): 62-91.

—_. 1999. Pavonia Cav. (Malvaceae). Flora Neotropica 76: 1-284.

—. 2009. A new species of Pavonia (Malvaceae) from the Atlantic coastal forests of eastern Brazil. Phytotaxa 2: 13-18.

KRAPOVICKAS, A. 1974. Acaulimalva, nuevo género de Malváceas. Darwiniana 19: 9-39.

- 1982. Novedades en Pavonia Cav. sect.Goetheoides Gürke (Malvaceae). Anais do XXXII Congresso Nacional de Botânica. Sociedade Botânica do Brasil. Universidade Federal do Piauí, Teresina: 67-84.

. 1990. Dos especies nuevas de Pavonia Cav, (Malvaceae) de Brasil. Anais do XXXVI Congresso Nacional de Botânica. Sociedade Botânica do Brasil, IBAMA, Brasilia, Brasil: 281-286.

—_. 2003. Sida sección Distichifolia (Monteiro) Krapov. comb. nov., stat nov. (MalvaceaeMalveae). Bonplandia 12 (1-4): 83-121.

—. 2006. Las especies argentinas y de países vecinos de Sida secc. Nelavaga (Malvaceae, Malveae). Bonplandia 15(1-2): 5-45.

. 2007. Novedades en el género Sida (Malvaceae, Malveae). Bonplandia 16(3-4): 193-208.

Original recibido el 20 de mayo de 2010; aceptado el 1 de julio de 2010. 
BONPLANDIA 19(1). 2010 